\title{
A High Resolution Atomic Force Microscopy Study of Poly(lactic acid-co-ethylene glycol)
}

\author{
Simon L. McGurk, Giles H.W. SAnders, Martyn C. Davies, Stanley S. Davis, * Lisbeth Illum, ${ }^{*}$ \\ Clive J. Roberts, ${ }^{\dagger}$ Snjezana STOLNIK, ${ }^{*}$ Saul J.B. TENDLER, \\ and Phil M. Williams
}
Laboratory of Biophysics and Surface Analysis, School of Pharmaceutical Sciences, The University of Nottingham, Nottingham, NG7 2RD, U.K. *Advanced Drug Delivery Group, School of Pharmaceutical Sciences, The University of Nottingham, Nottingham, NG7 2RD, U.K.

(Received September 20, 1999)

KEY WORDS Atomic Force Microscopy / Lateral Force / Poly(ethylene glycol) / Polymer brush /

In order to increase the systemic lifetime of drug delivery devices in the blood, polymers such as poly(propylene oxide-co-ethylene oxide) (PPO-PEO) and poly(lactic acid-co-ethylene oxide) (PLA-PEO) have been developed to enhance the resilience of surfaces to opsonization. ${ }^{1-7}$ These polymers incorporate segments that are relatively hydrophilic (PEO) and hydrophobic (PLA). Here we form a thin film of an amphipathic diblock copolymer of PLA and PEG (poly(ethylene glycol)) of monomeric ratio 6:5 by casting from chloroform. We have shown using X-ray photoelectron spectroscopy that both PLA and PEG molecules are present at the surface of such a film ${ }^{8}$ and by using surface plasmon resonance that the PEG portion when exposed to an aqueous environment extends into the liquid, masking the hydrophobic PLA portion. ${ }^{8-10}$ This phenomenon can be exploited to create steric barriers at interfaces, for example to resist protein adsorption. ${ }^{9}$ For such a polymer film it is important to understand the structure which the film adopts in an aqueous environment. Here we utilise atomic force microscopy (AFM) to investigate the surface structure and orientational characteristics of the PLA : PEG when exposed to aqueous conditions.

Theoretical studies and computer simulations ${ }^{11-13}$ predict that at high surface coverage and in the presence of an aqueous environment it is likely that the PEG chains will stretch out in the direction normal to the surface in structures termed polymer brushes. Previous AFM studies of similar systems ${ }^{14-16}$ include Lea et al. ${ }^{15}$ who have used force-distance measurements to investigate compression of PEG chains grafted onto silicon nitride surfaces and Iwata et $a .^{16}$ who have visualised the behavior of poly(acyrilic acid) (PAA) chains grafted onto a nucleopore membrane. By observing the topography of the surface at differing $\mathrm{pH}$, they inferred conformational changes of the grafted PAA. At low $\mathrm{pH}$ the underlying membrane pore contours were seen as the chains were precipitated on the membrane surface but at high $\mathrm{pH}$ the pores were filled with solvated grafted chains.

\section{EXPERIMENTAL}

\section{Polymer Film Preparation}

Films of PLA-PEG copolymer (PLA 6KDa and PEG $5 \mathrm{KDa}$ in a $6: 5$ ratio synthesised by Zeneca Pharmaceuticals, Alderley Park, Macclesfield, U.K.) were prepared from a $1.0 \% \mathrm{w} / \mathrm{v}$ solution in $\mathrm{CHCl}_{3}$ (Fisons Scientific Equipment, Loughborough, U.K.). A $50 \mu$ l aliquot of the respective solution was dropped onto the silver surface of a surface plasmon resonance (SPR) slide, which had been previously cleaned with acetone (Fisons Scientific Equipment, Loughborough, U.K.), rotating at approximately $2000 \mathrm{rpm}$ creating a thin continuous film (confirmed by AFM and SPR, data not shown). The silver slides (Ortho Clinical Diagnostics, Chalfont St. Giles, U. $K$.) were used as a substrate to relate the experimental data to comparative SPR studies. ${ }^{10}$ SPR is an in situ optical technique which is typically employed to provide kinetic information on interfacial events such as deposition, hydration and degradation. ${ }^{17}$

\section{Atomic Force Microscopy}

$\mathrm{AFM}^{18}$ was undertaken in an aqueous environment using a Thermomicroscopes Explorer (Thermomicroscopes Corp., Bicester, U.K.). Commercially obtained (Thermomicroscopes) tips were used. All data presented was recorded during the period with tip scanning left to right. The commercial control software allowed for lateral force, error mode and topographical images to be obtained concurrently. For higher resolution scans error mode and lateral force signals ${ }^{19}$ were used with the feedback loop disabled. This mode of operation provided better resolution of images for this sample in comparison to the conventional mode of acquisition of topography. A discussion of the advantage of lateral force imaging of the sample surface is included later. In the case of high resolution images (Figures 2a and 3) data sets were taken at a variety of scan speeds and rotation angles to ensure that the features seen were not artefacts arising from background noise.

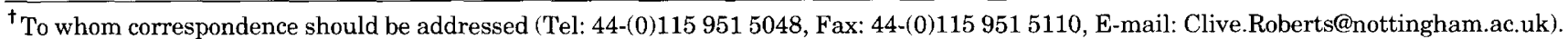



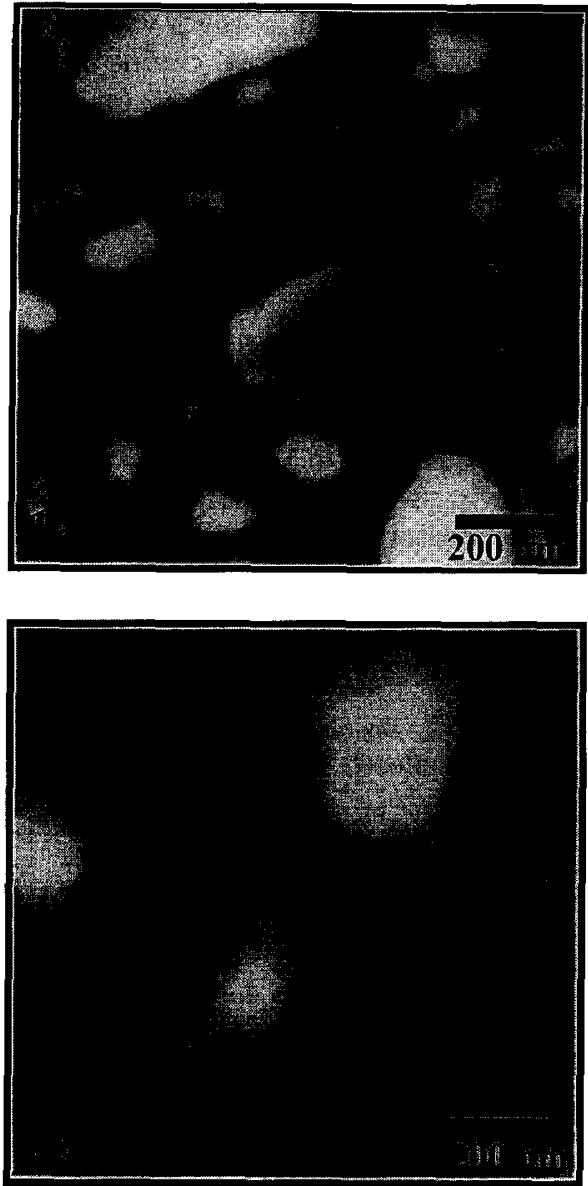

Figure 1. AFM image of the silver SPR substrate prior to (1a) and after (1b) polymer treatment (z scale $30 \mathrm{~nm}$, white regions being higher).

\section{RESULTS AND DISCUSSION}

Figure 1a shows a $1 \mu \mathrm{m}$ square topographical image of an uncoated silver surface in $\mathrm{pH} 7.4$ phosphate buffer solution. Comparison to Figure 1b, which shows a similar sized area of the PLA-PEG $(6: 5)$ polymer film, confirms that the polymer coating is extremely smooth, uniform and homogeneous and that the majority of the features seen in Figure $1 \mathrm{~b}$ are a result of the gross topography of the underlying silver. Lateral force imaging of the uncoated silver substrate reveals no detail (data not shown) apart from topography induced contrast commonly associated with relatively rough surfaces.

In Figure 2a, a $40 \mathrm{~nm} \times 40 \mathrm{~nm}$ area of the PLA-PEG film surface was imaged in lateral force mode. The lateral force image shows individual features of between $0.5 \mathrm{~nm}$ to $1 \mathrm{~nm}$ in size, which is consistent with the molecular dimensions of the apex of the individual PEG chains tightly packed in polymer brushes emanating from the polymer surface. It is important to distinguish these results from those obtained from equilibrium adsorption studies of similar polymers from aqueous solution, where surface densities of the PEG chains are an order of magnitude lower than that observed for this study. ${ }^{20}$

The lateral force AFM data provide better resolution in comparison with other imaging modes due the nature (a)

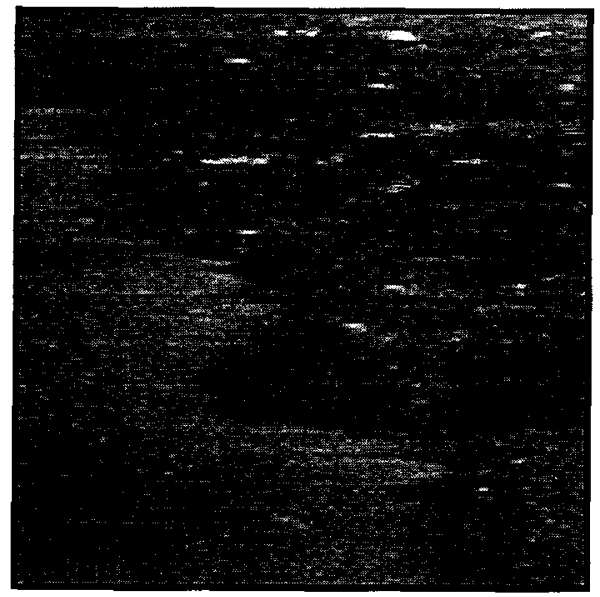

(b)
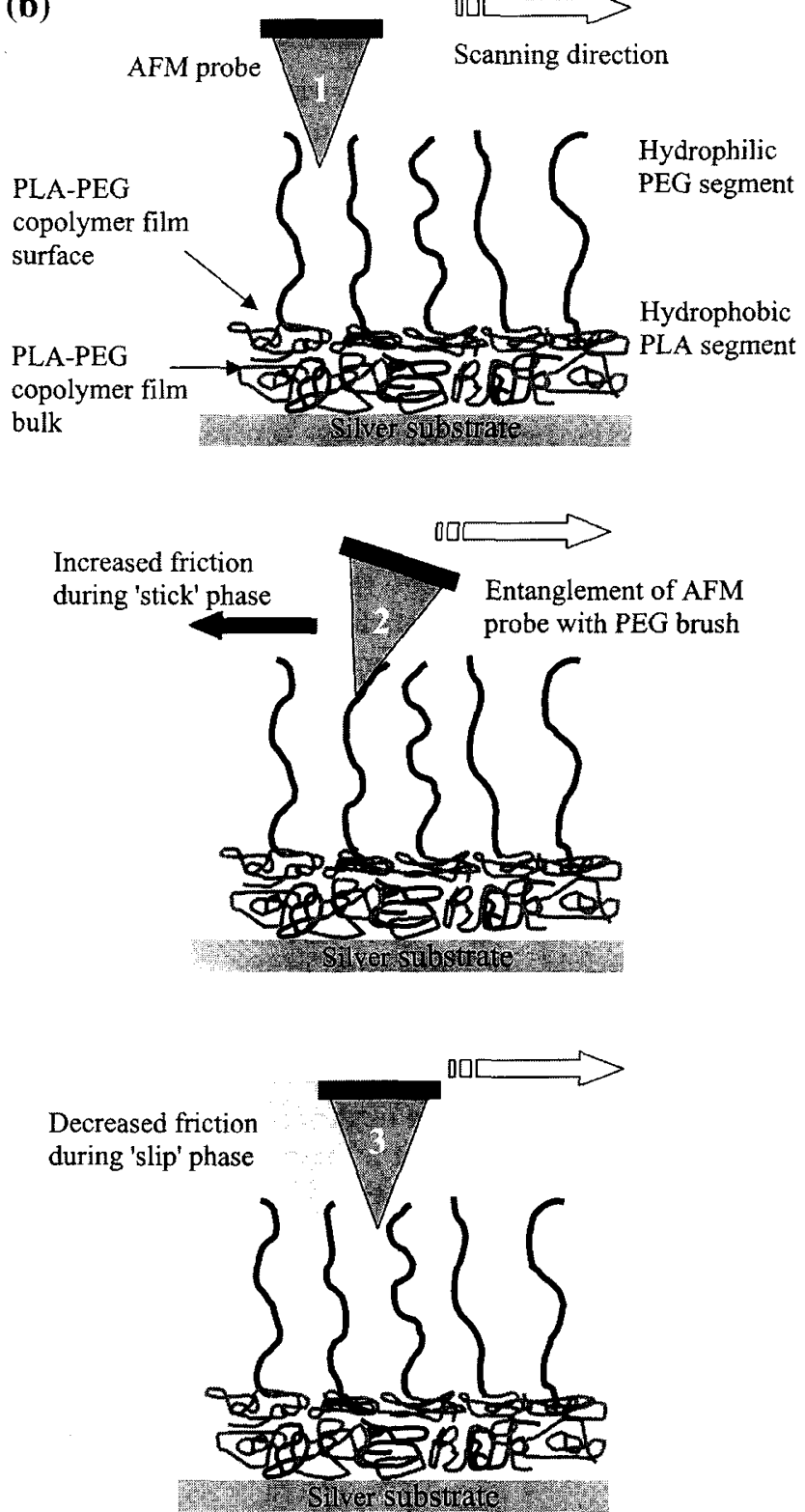

Figure 2. (a) A $40 \mathrm{~nm} \times 40 \mathrm{~nm}$ lateral force scan of the PLA-PEG copolymer showing the individual features arising from the apex of the PEG brushes. (b) Schematic of interaction between tip and PEG brushes, showing how the friction produced by individual PEG chains induces movement in the AFM cantilever. 


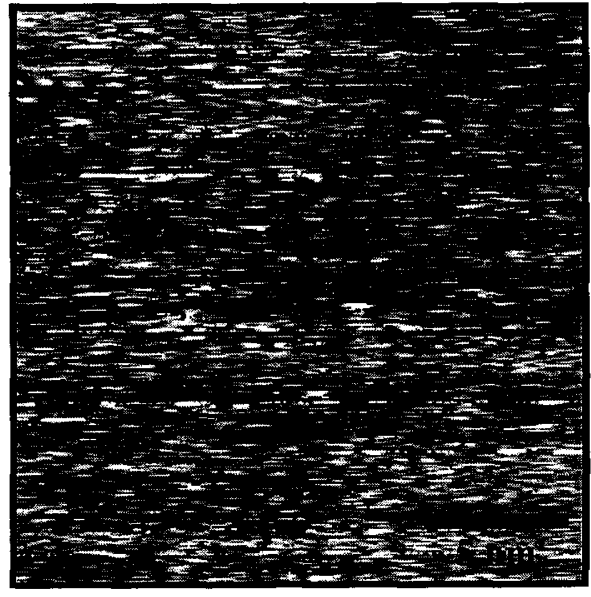

Figure 3. A $20 \mathrm{~nm} \times 20 \mathrm{~nm}$ lateral force scan showing features related to the apex of the PEG brush emanating from the silver substrate.

of the signal measured. A topographical image arises from deflection of the cantilever in a direction normal to the polymer surface (and parallel to the PEG chain orientation) whereas the lateral force image is formed from the deflection of the cantilever normal to the polymer chains. During imaging the tip is likely to contact not merely the top of the PEG chains but also some of the underlying chain, illustrated in Figure $2 \mathrm{~b}$. This produces a force in the lateral direction of the tip but not in the vertical direction, and hence, image contrast is increased in the lateral force mode. Similar observations have been made for a number of materials, such as layered dichalcogenide tungsten diselenide ${ }^{21}$ and poly(tetrafluoroethylene) $^{22}$ where the imaging mechanism is proposed to result from a 'stick-slip' motion of the AFM probe, and this is a likely explanation here also for the enhanced resolution observed. This hypothesis also explains why the polymer chains are detected when considering that thermal motion of the chains would be expected to 'smear-out' any such contrast as would most likely occur with standard topographic AFM imaging.

Figure 3 displays a $20 \mathrm{~nm}$ square lateral force image of the same area. In this case the ends of the single PEG chains are visible. An elongation of the features can be seen in the scan direction, which may be explained from the tip-sample interaction. The tip draws the polymer chains from the equilibrium position to an extended state until moving onto the next polymer chain. This indicates that single chains of a hydrated copolymer have been directly imaged rather than indirectly imaged ${ }^{16}$ as before. An average of approximately three PEG chains per $\mathrm{nm}^{2}$ can be seen in the image. This corresponds to a packing density of $5 \times 10^{-6} \mathrm{~mol} \mathrm{~m}^{-2}$.

\section{SUMMARY}

We have utilised in situ AFM in lateral force mode to directly image topographical features which, we propose are the apex of the individual PEG chains extending into the aqueous environment from the surface. This corresponds to in vitro visualisation of polymer brushes at the molecular level. These data are consistent with models proposed for the structure of such films and the mechanisms by which they are believed to resist protein interactions.

Acknowledgments. The authors would like to thank S. Purkiss and P. R. Gellert of Zeneca Pharmaceuticals for the polymers utilised in this study. SLM thanks the BBSRC for providing his studentship. GHWS thanks the BBSRC for postdoctoral funding. SS is an Astra Charnwood lecturer.

\section{REFERENCES}

1. T. Verrecchia, G. Spenlehauer, D. V. Bazile, A. MurrayBrelier, Y. Archimbaud, and M. Veillard, Controlled Release, 36, 49 (1995).

2. D. F. Williams and S. P. Zhong, Int. Biodeterioration and Biodegradation, 34, 95 (1994).

3. R. J. Green, S. Tasker, J. Davies, M. C. Davies, C. J. Roberts, and S. J. B., Tendler, Langmuir, 13, 6510 (1997).

4. B. E. Rabinow, Y. S. Ding, C. Qin, M. L. McHalsky, J. H. Schnieder, K. A. Ashline, T. L. Shelbourn, and R. M. Albrecht, J. Biomater. Sci. Polym. Ed., 6, 91 (1994).

5. S. I. Jeon, J. H. Lee, J. D. Andrade, and P. G. De Gennes, Colloid Interface Sci., 142, 1 (1991).

6. P. Ferruti, P. Penco, P. D'Addato, E. Ranucci, and R. Deghenghi, Biomaterials, 16, 1423 (1995).

7. W. J. Feast, H. S. Munro, and R. W. Richards, "Polymer Surface and Interfaces II", M. C. Davies, Ed., John Wiley and Sons, England, 1993, Chap. 8, p 204.

8. F. E. Black, M. Hartshorne, M. C. Davies, C. J. Roberts, S. J. B. Tendler, P. M. Williams, K. M. Shakesheff, S. M. Cannizzaro, and R. Langer, Langmuir, 15, 3157 (1999).

9. N. Patel, Thesis in The University of Nottingham, 1998.

10. N. Patel, R. Bhandari, K. M. Shakesheff, S. M. Cannizzaro, M. C. Davies, R. Langer, C. J. Roberts, S. J. B. Tendler, and P. M. Williams, submitted to Langmuir, 15, 7252 (1999).

11. S. Alexander, J. Phys. (Paris), 38, 977 (1977)

12. P. G. de Gennes, Macromolecules, 13, 1069 (1980).

13. A. Chakrabarti and R. Toral, Macromolecules, 23, 2016 (1990).

14. S. L. S. Stipp, Langmuir, 12, 1884 (1996).

15. A. S. Lea, J. D. Andrade, and V. Hlady, Colloids and Surfaces A, 93, 349 (1994).

16. H. Iwata, I. Hirata, and Y. Ikada, Langmuir, 13, 3063 (1997).

17. J. Homola, S. S. Yee, and G. Gauglitz, Sensors and Actuators B-Chemical, 54, 3 (1999).

18. H. G. Hansma, K. J. Kim, D. E. Laney, R. A. Garcia, M. Argaman, M. J. Allen, and S. M. Parsons, J. Struct. Biol., 119, 99 (1999).

19. C. M. Mate, G. M. McCelland, R. Erlandsson, and S. Chaing, Phys. Rev. Lett., 59, 1942 (1987).

20. S. Stolnik, N. C Felumb, C. R Heald, M. C. Garnett, L. Illum, and S. S. Davis, Colloids and Surfaces A, 122, 151 (1997).

21. T. Schimmel, J. Kuppers, and M. Luxsteiner, Thin Solid Films, 264, 212 (1995)

22. G. J. Vancso, S. Forster, and H. Leist, Macromolecules, 29, 2158(1996). 